\title{
Anterior Segment Imaging and Treatment of a Case with Syndrome of Ectopia Lentis, Spontaneous Filtering Blebs, and Craniofacial Dysmorphism
}

\author{
Ahmad M. Mansour ${ }^{a}$ b Mohammad H. Younis ${ }^{a, b}$ Rola H. Dakroub ${ }^{a, b}$ \\ ${ }^{a}$ Department of Ophthalmology, American University of Beirut, and ${ }^{b}$ Department of \\ Ophthalmology, Rafic Hariri University Hospital, Beirut, Lebanon
}

\section{Key Words}

Anterior segment imaging · Ectopia lentis · Spontaneous filtering blebs · Craniofacial dysmorphism

\begin{abstract}
Purpose: To report the ultrasound biomicroscopy (UBM) and surgical findings in a subject with a syndrome of ectopia lentis, spontaneous filtering blebs, and craniofacial dysmorphism (Traboulsi syndrome). Methods: Case report, using a 40-MHz UBM wide-field anterior segment scan and anterior segment optical coherence tomography (OCT). Results: A 16year-old orphan girl presented with visual loss to the level of 6/60 (20/200) bilaterally. She had a central corneal opacification with retrocorneal fibrosis. The anterior chamber was flat with a very poorly dilating pupil. The lens was central in location. Perilimbal conjunctival blebs were bilateral with an intraocular pressure of $8 \mathrm{~mm} \mathrm{Hg}$. UBM and anterior segment OCT revealed chronic apposition of the iris to the cornea with angle closure, delineation of the bleb tract and rarefaction of the zonules. The girl had abnormal facial features (a beaked nose and long face) with normal chromosomal studies, negative fluorescent in situ hybridization study for velocardiofacial syndrome and an absence of signs suggesting Marfan syndrome. Under general anesthesia, attempts at deepening the anterior chamber with sodium hyaluronate $3 \%$ led to a spontaneous dislocation of the lens into the anterior chamber, facilitating its aspiration. Deepening of the angle was found after lens removal. Retrocorneal fibrosis persisted after surgery, but the bleb height decreased. Best corrected visual acuity did not improve from the preoperative level beyond 6/60 (20/200) because of central retrocorneal fibrosis. Conclusions: Early surgical removal of the lens is necessary in
\end{abstract}

Ahmad M. Mansour, MD

Department of Ophthalmology

American University of Beirut

PO Box, Beirut 1136044 (Lebanon)

E-Mail ammansourmd@gmail.com 
this syndrome to avoid irreversible corneal and trabecular meshwork damage in chronic apposition of the iris to the cornea. UBM can help in the delineation of the bleb tract and document resolution of angle closure after surgery.

\section{Introduction}

Inadvertent bleb may form as a result of a fistula which allows aqueous to flow from the anterior chamber into the subconjunctival space. Most conjunctival blebs follow episodes of scleritis [1], accidental penetrating injury or ocular surgical procedures (cataract surgery, scleral fixated intraocular lens implantation, scleral tunnel lensectomy, and cyclophotocoagulation). Spontaneous filtering blebs are rare [2] and have been observed with few systemic disorders (scleroderma [3]), ocular abnormalities (Terrien's marginal degeneration [4] and Axenfeld syndrome [5]), or with systemic conditions, such as familial craniofacial dysmorphism with spontaneous bleb formation $[6,7]$ known in Lebanon as Traboulsi syndrome. We present the anterior segment imaging and treatment in a case that provides new insights into the pathophysiology of Traboulsi syndrome.

\section{Case Report}

A 16-year-old female orphan (fig. 1) has been complaining of bilateral visual loss for several years. Both uncorrected and corrected (with $-7.0 \mathrm{dpt}$ ) visual acuities were 6/60 $(20 / 200)$ bilaterally. She had a central superficial corneal opacification as well as central retrocorneal nodular thickening bilaterally. She also had iridocorneal touch with a diffusely flat anterior chamber (fig. 2). The pupil failed to dilate from diffuse posterior synechiae. The lens was in a central location bilaterally. Conjunctival blebs were evident superiorly and nasally (fig. 2) in both eyes with an intraocular pressure of $8 \mathrm{~mm} \mathrm{Hg}$. The posterior pole could be visualized in the left eye with difficulty using a 90-dpt lens and revealing a cup-todisc ratio of 0.1. Using ultrasonography, the axial length measured $19.41 \mathrm{~mm}$ in the right eye and $20.12 \mathrm{~mm}$ in the left eye. The cornea was visualized using anterior optical coherence tomography (OCT) (Visante OCT; Carl Zeiss Meditec Inc., Jena, Germany) with good delineation of the central retrocorneal fibrosis (fig. 3). Near apposition of the cornea to the iris was clearly demonstrated with angle closure (fig. 4). Few zonules were delineated. Besides the apposition of the iris to the cornea with angle closure, both rarefaction of the zonules and bleb tracts were more clearly imaged by ultrasound biomicroscopy (UBM) using a 40-MHz UBM probe (Eye cubed Ellex; Ellex Innovative Imaging Inc., Sacramento, Calif., USA). Under general anesthesia, attempts at deepening the anterior chamber with sodium hyaluronate $3 \%$ led to spontaneous dislocation of the lens into the anterior chamber (fig. 2), facilitating its aspiration (fig. 2). Deepening of the angle was somewhat more evident on UBM than anterior segment OCT after lens removal (fig. 5). Retrocorneal scar tissue persisted after surgery (fig. 3), but the bleb height decreased clinically and by UBM (comparing to the fellow eye) after surgery (fig. 5). Best corrected visual acuity did not improve from the preoperative level beyond 6/60 (20/200) partly from the central retrocorneal scar (fig. 3). The patient declined a second eye surgery to remove the subluxated lens after 4 years of follow-up.

The patient had an elongated face and beaked nose (fig. 1). She had some features suggestive of velocardiofacial syndrome like a prominent broad nose [8]. Genetic consultation ruled out both homocystinuria (normal serum amino acid quantitation) and DiGeorge 
Mansour et al.: Anterior Segment Imaging and Treatment of a Case with Syndrome of Ectopia Lentis, Spontaneous Filtering Blebs, and Craniofacial Dysmorphism

syndrome (no deletion in the region 22q11.2 by fluorescent in situ hybridization), and confirmed a normal karyotype.

\section{Discussion}

Traboulsi and colleagues [6] described 6 members of a family with a syndrome of mild facial dysmorphism, subluxation of the crystalline lenses, variable degrees of angle closure with iridocorneal adhesions, patchy iris atrophy, and scleral thinning. Three non-operated eyes of two patients had spontaneous filtering blebs that presented as avascular cystic elevations of the superior conjunctiva. Systemic work-up of all patients was negative for evidence of diseases known to be associated with dislocated lenses. Furthermore, Traboulsi and colleagues [7], subsequently reported 4 members of a Lebanese Druze family with the syndrome of lens dislocation, spontaneous filtering blebs, scleral thinning, anterior segment abnormalities and a distinctive facial appearance not compatible with Marfan syndrome (negative echocardiogram), but with autosomal recessive inheritance. The absence of scleral thinning in the current case may relate to the short eye status hence a thickened sclera. The formation of spontaneous filtering blebs in reported cases of Traboulsi syndrome [6, 7] may relate to the scleral thinning. Scleral thinning occurs in progressive myopia as in the family described by Dagi and Walton [9]. A posterior channel of communication was apparent on UBM between the bleb and ciliary body similar to the findings in a traumatic bleb as described by Khouri et al. [2].

The current syndrome is different from the syndrome described by Dagi and Walton [9] who described the clinical features of an atypical presentation of ectopia lentis consisting of primary anterior axial lens subluxation in childhood, associated progressive myopia, and complicating angle-closure glaucoma but without bleb formation. This clinical triad consists of rapidly increasing myopia, normal axial length, and progressive axial subluxation. Prophylactic lensectomy provides a safe and sight-saving treatment that arrests and, at times, reverses the progression of angle-closure glaucoma. Ectopia lentis with anterior axial subluxation and progressive myopia can be a sight-threatening condition when significant forward mobilization of the lens results in synechial angle-closure glaucoma. Recognition of this clinical entity can expedite diagnosis and prevent irreversible loss of vision secondary to glaucoma. Because the primary mechanism of angle closure is angle crowding, peripheral iridotomy does little to control the rise in intraocular pressure. According to Dagi and Walton [9], lensectomy appears to represent the most definitive treatment in this syndrome; and lensectomy was ultimately required to halt progressive angle closure resulting from relentless axial subluxation [9]. Similarly, peripheral iridotomy may be of little value in our case and our therapy is to restore the angle anatomy by lens removal. Our patient had a small eye, low intraocular pressure and spontaneous bleb. We hypothesize that uveal effusion in a nanophthalmic or small eye led to angle apposition with anterior segment ischemia resulting in zonular damage and secondary lens subluxation. Alternative explanations include: (1) primary cleft with secondary forward iris movement and corneal iris apposition and (2) a primary collagen synthesis problem with subsequent scleral melt, bleb formation, and secondary forward iris movement.

Congenital or heritable dislocation of the lens results primarily from abnormal zonules. Weakening, stretching, or breakage of the zonules frees the lens to migrate and sublux. Ectopia lentis occurs in Marfan syndrome as well as in a large number of other inheritable disorders (homocystinuria, Weill-Marchesani syndrome, ectopia pupillae, microsphero- 
Mansour et al.: Anterior Segment Imaging and Treatment of a Case with Syndrome of Ectopia Lentis, Spontaneous Filtering Blebs, and Craniofacial Dysmorphism

phakia, mandibulofacial dysostosis, etc.). It can occur as an isolated abnormality, secondary to ocular trauma or syphilis.

Myopia in the presence of ectopia lentis in Marfan syndrome may be due to axial elongation of the eye. In addition, two lenticular mechanisms may also contribute to the myopia, and we suspect they are its cause in the present case: an anterior shift of the lens-iris diaphragm moving the focal point of the eye very anteriorly, as well as an antero-posterior thickening of the lens (spherophakia-like) [9].

Also, Peters' anomaly is a possibility in the differential diagnosis of central opacity of the cornea with retrocorneal fibrous tissue and with iridocorneal synechiae [10]. Peters' anomaly is characterized by central corneal opacity (leukoma), thinning of the posterior aspect of the cornea, iridocorneal adhesions, and keratolenticular adhesion or cataract. The presence of lens abnormalities in Peters' anomaly is more frequently associated with systemic anomalies such as a cleft lip and palate, short stature, broad hands and feet, and variable mental delay. These were not present in the case described in this report.

We presented additional insight into the Traboulsi syndrome of facial dysmorphism with spontaneous conjunctival blebs using anterior segment OCT and UBM technologies. Early lensectomy seems indicated to prevent irreversible corneal scarring and angle damage from chronic apposition of the iris to the cornea.

\section{References}

1 Mantravadi AV, Stock EL: Spontaneous filtration bleb as a consequence of scleritis. Arch Ophthalmol 2007;125:1578-1579.

-2 Khouri AS, Newman FR, Fechtner RD: Ultrasound biomicroscopy demonstrating etiology of a spontaneous filtering bleb. Eye 2006;20:1441-1442.

-3 Gerke E, Meyer-Schwickerath G, Joussen F: Sclerodermie en coup de sabre mit spontaner Filterkissenbildung. Klin Monbl Augenheilkd 1976;168:426-428.

-4 Wong VA, Beiko G, Pavlin CJ, Rootman DS: Treatment of a spontaneous filtering bleb due to Terrien's marginal degeneration with injection of autologous blood. Can J Ophthalmol 1995;30:377-379.

-5 Nemet P, Bracha R, Lazar M: Spontaneous filtering blebs in Axenfeld syndrome. Am J Ophthalmol 1973;76:590-591.

-6 Shawaf S, Noureddin B, Khouri A, Traboulsi EI: A family with a syndrome of ectopia lentis, spontaneous filtering blebs, and craniofacial dysmorphism. Ophthalmic Genet 1995;16:163-169.

7 Haddad R, Uwaydat S, Dakroub R, Traboulsi EI: Confirmation of the autosomal recessive syndrome of ectopia lentis and distinctive craniofacial appearance. Am J Med Genet 2001;99:185-189.

-8 Mansour AM, Goldberg RB, Wang FM, Shprintzen RJ: Ocular findings in the velo-cardio-facial syndrome. J Pediatr Ophthalmol Strabismus 1987;24:263-266.

19 Dagi LR, Walton DS: Anterior axial lens subluxation, progressive myopia, and angle-closure glaucoma: recognition and treatment of atypical presentation of ectopia lentis. J AAPOS 2006;10:345-350.

-10 Hwang DK, Yang AH, Yen MY, Wang AG: Ultrastructure of anterior lens capsule in Peters' plus syndrome. Eye 2007;21:862-864.

The authors have no financial interests. This case report was presented at the 2012 Second World Congress of Pediatric Ophthalmology and Strabismus, Milan, Italy. 
Mansour et al.: Anterior Segment Imaging and Treatment of a Case with Syndrome of

Ectopia Lentis, Spontaneous Filtering Blebs, and Craniofacial Dysmorphism

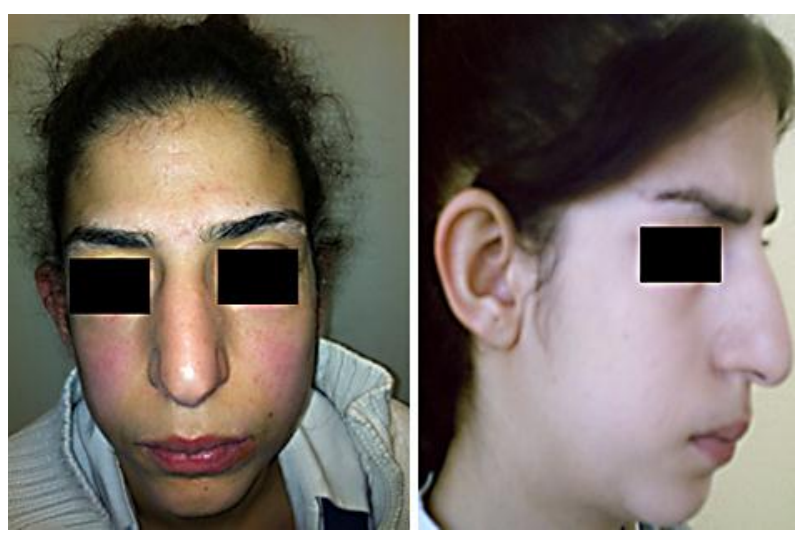

Fig. 1. Right: facial dysmorphism includes an elongated face with prominent broad nose. Left: side view of the face shows a beaked nose.
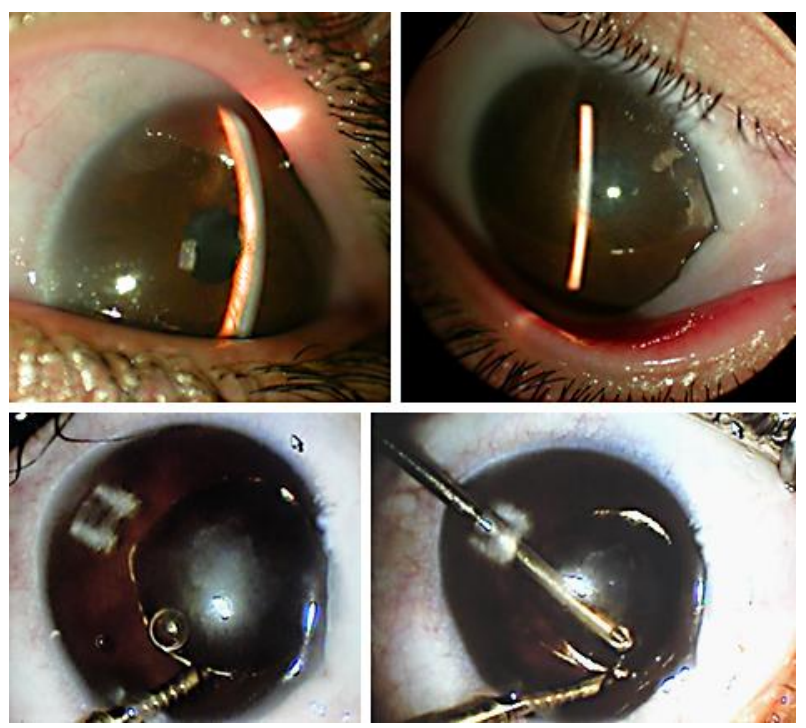

Fig. 2. Top left: slit lamp photography of the anterior segment of the left eye. The slit passes by the edge of the miotic pupil after dilation. Note the flat anterior chamber. Top right: slit lamp photography of the anterior segment of the right eye shows prominent nasal bleb, flat anterior chamber and central superficial corneal opacity. Bottom left: slit lamp photography of the anterior segment of the right eye shows prominent nasal bleb, flat anterior chamber and central superficial corneal opacity. Bottom right: following injection of hyaluronic acid to deepen the anterior chamber in the right eye, there was a sudden appearance of the clear lens into the anterior chamber. Note the central corneal scar. 


\section{Case Reports in Ophthalmology}

\begin{tabular}{l|l}
\hline Case Rep Ophthalmol 2013;4:84-90 \\
\hline DOI: 10.1159/000350951 & $\begin{array}{l}\text { ○ 2013 S. Karger AG, Basel } \\
\text { www.karger.com/cop }\end{array}$ \\
\hline
\end{tabular}

Mansour et al:: Anterior Segment Imaging and Treatment of a Case with Syndrome of Ectopia Lentis, Spontaneous Filtering Blebs, and Craniofacial Dysmorphism

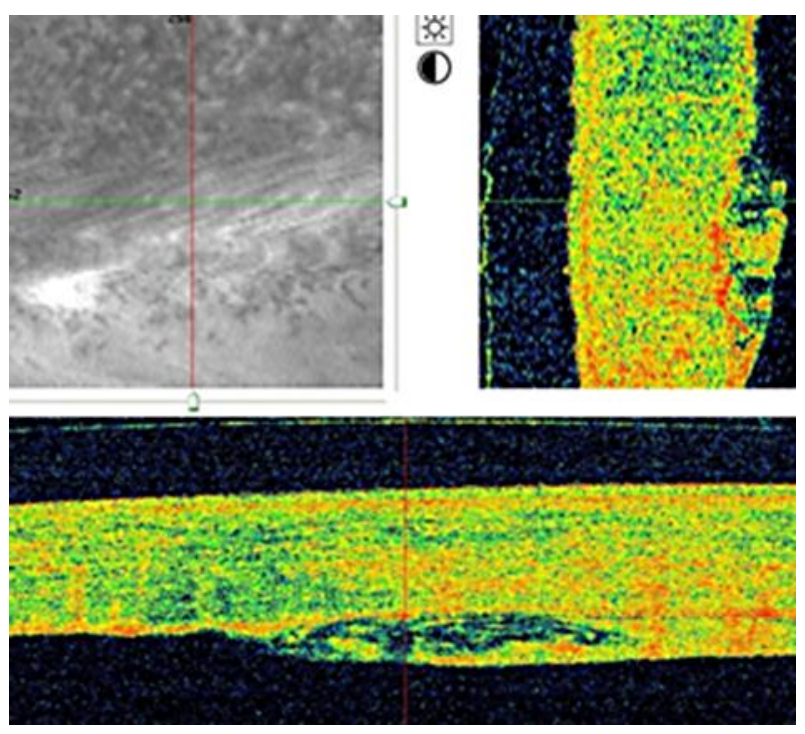

Fig. 3. Anterior segment OCT scans show central retrocorneal fibrosis in the right eye by vertical scan (top right) and horizontal scan (bottom).

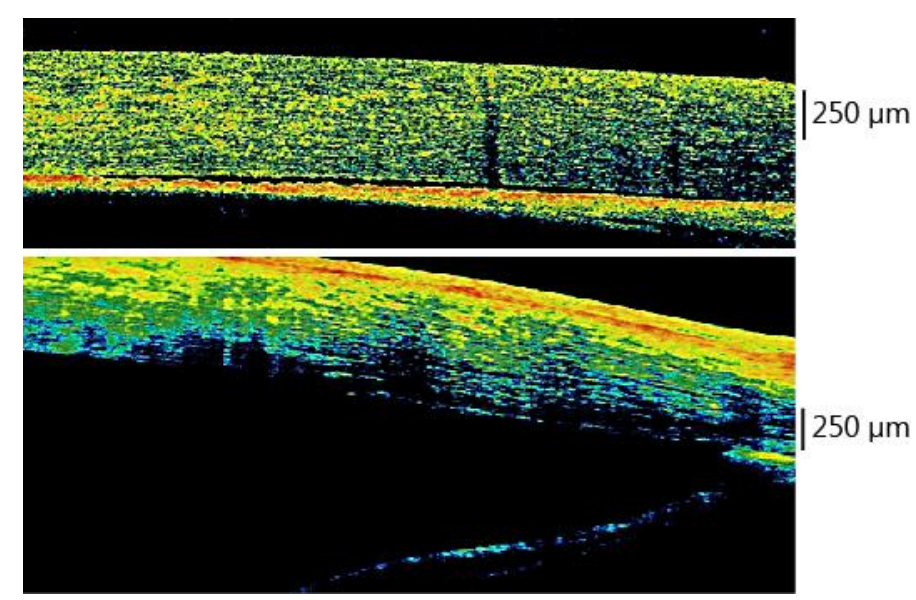

Fig. 4. Top: by anterior OCT, the midperiphery of the left cornea shows angle closure, a flat anterior chamber $(16 \mu \mathrm{m})$ and a peripheral corneal thickness of $625 \mu \mathrm{m}$. Bottom: anterior OCT of the angle after surgery to the right eye shows resolution of angle closure configuration. 


\begin{tabular}{l|l}
\hline Case Rep Ophthalmol 2013;4:84-90 \\
\hline DOI: $10.1159 / 000350951$ & $\begin{array}{l}\text { ○ 2013 S. Karger AG, Basel } \\
\text { www.karger.com/cop }\end{array}$ \\
\hline
\end{tabular}

Mansour et al.: Anterior Segment Imaging and Treatment of a Case with Syndrome of

Ectopia Lentis, Spontaneous Filtering Blebs, and Craniofacial Dysmorphism
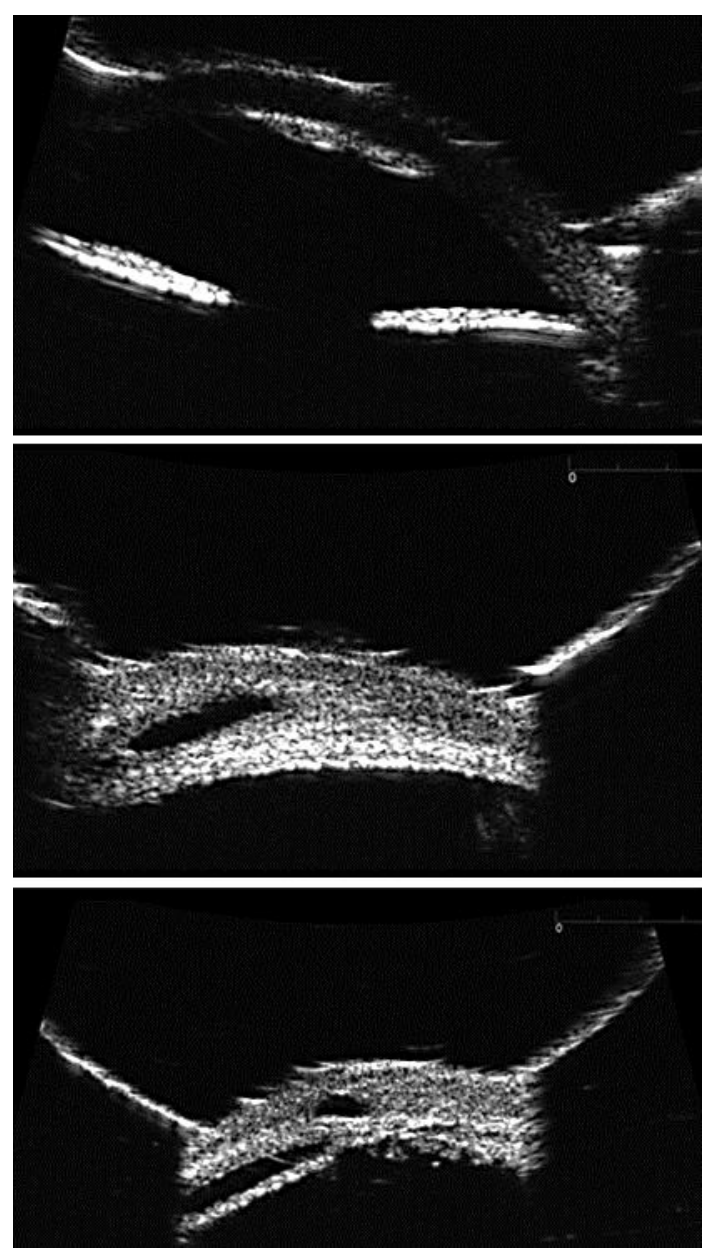

Fig. 5. Top: UBM of the right eye after surgery shows the restoration of the normal angle anatomy as well as echogenic central retrocorneal fibrosis. UBM of the left unoperated eye (middle panel) shows a large fistula, while the operated right eye (bottom panel) shows a small fistula. 